\title{
Light Cones as Quantum Phenomena
}

\author{
Terry B. Bollinger (1) 1 \\ ${ }^{1}$ Apabistia Research, Ashburn, VA, USA 20147
}

(Incepted Tuesday November 2021; Published Tuesday November 2021; Tweaked 2021-11-18.09:44)*

\begin{abstract}
Quantum erasure experiments push the boundary between the quantum and classical worlds by letting delayed events influence the state of previously recorded and potentially widely distributed classical information. The only significant restriction to such unsettling violations of forward-only causality is that the distribution of forward-dependent information cannot cross out of the light cone boundaries of the event in the past, a feature that ensures no violations of causality — no rewriting of anyone else's recorded histories - can occur. The erasure interpretation of this conundrum requires rewriting of information recorded and distributed in the past, which would itself be a violation of causality. The quantum predestination interpretation removes the causal rewriting issue. However, quantum predestination requires detailed coordination of inputs from outside of the forward-dependent event's light cone, thus massively violating the same limit that prevents causality violations in such events. Yet another approach is to invoke the Schrödinger's cat variant of quantum erasure in which arbitrarily complex classical events within the light cone become quantum dependent upon the future event. As with all Schrödinger's cat interpretations of quantum mechanics, this variant of quantum erasure violates causality by discarding local classical histories such as the information-rich state of the cat's body. The most straightforward interpretation of erasure experiments is to follow the lead of the equations themselves, which transform on paper as if their components are independent of ordinary space and time limits, up to the limits imposed on them by the speed of light. Interpreting the light cone of each quantum system as an atemporal, aspatial unit in which classical time and space have no meaning results in a multi-scale, matter-dependent definition of spacetime in which every light cone is a singular quantum entity. In such a universe, both time and space are defined not as pre-existing, mass-independent continuums but as the consensus of vast numbers of constantly interacting and mutually limiting quantum-entity light cones.
\end{abstract}

DOI: https://doi.org/10.48034/20211109

\section{INTRODUCTION}

Hossenfelder [1] noted that most interpretations of delayed-choice quantum erasure experiments fail to consider the pre-existence of the final "changed" result in data already classically recorded and distributed. One can philosophically and even mathematically disregard the importance of such historical results as, for example, artifacts that become parts of parallel universes after erasure occurs. While fascinating to discuss, such approaches cannot easily be classified as scientific since they discard the very historical records upon which the

Published by Apabistia Press under the Creative Commons Attribution 4.0 International License. You are free to redistribute this article as you choose, provided only that you preserve the author attribution, article title, journal citation, and DOI.

${ }^{*}$ This document uses Kenobi versioning. The online name always points to the most recent document version, while obl, ob2,... suffixes indicate obsolete releases of the document.
This is about: Quantum erasure, Quantum predestination, Retrocausality, Quantum interpretation scientific method relies, and since by discarding such data, they are no longer disprovable.

Thus Hossenfelder describes the delayed choice erasure conundrum in its sharpest terms: Given solid experimental evidence [2] for the existence of devices in which events with earlier classically recorded timestamps depend on events with later timestamps, what interpretation gives the best conceptual and analytical models of such devices?

\section{QUANTUM PREDESTINATION}

In an earlier paper [3], the author proposed an interpretation more in keeping with the sequence of events seen in experiments: quantum predestination. If earlier events exist as recorded classical data with potentially arbitrarily vast distribution, e.g., via the internet, the later outcome of the experiment must, in some sense, be predestined or superdetermined [4] at that earlier time.

From a modeling perspective, the highest-value feature of quantum predestination is that it avoids discarding the idea that classical history, information, and causality are 
all quite real and invariant over time. One can, of course, abandon this position, both interpretively and, in the case of the many-world interpretation [5], with considerable and often delightfully appealing mathematical detail. Unfortunately, denying the permanence of recorded history also undermines the scientific method, which requires the reproducibility of past results. Furthermore, any theory that dismisses causality cannot be falsifiable since it undermines the question of what "false" means.

The phrase quantum predestination avoids at least this trap particular trap by doing nothing more than following through on the implications of Hossenfelder's pointed observation: Data that gets recorded, data stays recorded.

\section{THE PREDESTINATION PROBLEM}

Quantum predestination, however, has difficulties of its own. The most conspicuous of these is that it undermines the very limitation that most conspicuously keeps quantum erasure from undermining reality, which is that such events cannot distribute data outside the boundaries of their forward light cones. This vital limitation prevents paradoxes in which such information could, in principle, undermine or contradict a result that has already occurred.

Quantum predestination conflicts with this light cone limit because it assumes control over events outside its light cone. For example, a human experimenter or machine in the future must respond not just to events within the quantum predestination light cone but also to the enormous number of forward light cones touched by its backward light cone. An easily overlooked feature of quantum entanglement is that even though its non-locality impacts transcend light-speed limits when interpreted using continuum spacetime, the time required to set up an experimentally meaningful entanglement is very much subject to speed-of-light constraints. For example, neither a fermion's Schrödinger wave nor a photon's wavefront can expand classically in $x y z$ space faster than light speed, thus limiting their non-zero expectations to the light cone.

Since a quantum predestination event cannot influence any forward events outside of the light cone, there is no known mechanism in physics by which it could enlist the massive coordination needed to, for example, entice a human observer intent on random behavior to tweak polarizers just so to give, on average, the expected nonlocal correlations.

\section{THE COMPUTATION PROBLEM}

A more subtle point is worth noting at this point. Even if there existed a known method in physics by which quantum predetermining events could influence the other light cones that contribute to their final predestined results, there remains a massive computation problem. Physics has an equivalence to computation since it predicts specific outcomes for given sets of entities and states. Attached to this interpretation is a constraint: The less mass and energy available to any given calculation, the fewer the number of bits of historical information generated in the result.

This principle applies not just to classical physics but to quantum physics, where the uncertainty principle ensures the non-emergence of detailed historical records without massive additions of energy. Thus the light-mass electrons in orbitals around protons generate no classical data over time about their locations and momentums, other than the data represented expressed by the orbitals. This situation can, of course, change radically when a photon interacts with such an atom. But at that point, the sudden increase in local energy enables the emergence of more classical, recorded history about the electron and its state.

This simple principle can be discomforting in quantum computing. It shows up mainly under the moniker of error correction, meaning large ensembles with considerable total energies are required to arrive at the results hinted at by infinitely precise matrix representations. Peres may have hinted at this situation obliquely as far back as 1985 [6] when he noted in the last paragraph of a paper that "Ultimately, quantum computer making full use of a continuous logic may turn out to be more akin to an oldfashioned analog computer, rather than to a modern digital computer. This would indeed be an ironic twist of fate."

Peres suspected that the relationship between quantum and classical information was not sufficiently understood to ensure practical computation using low mass-energies. On the other hand, the very existence of organic molecules that bridge the boundary between classical structure and quantum indeterminacy [7] [8] suggests that energyefficient quantum computing at the molecular scale is possible, even if still not well understood.

The problem in the case of quantum predestination is that the total computational power required to coordinate and predestinate responses from an immense number of incoming light cones, even if there did exist known physics capable of accessing those cones, is staggering in scope and baffling in complexity. If the mass-energy available within the coordinating light cone limits the total computation available, it is difficult to see how sufficient physics-level computational resources are available, let alone sufficient coordinating guidance on how to apply them.

\section{THE CAT-STATE PROBLEM}

In the audio recordings of his 1963 lectures on physics, Feynman once noted that [9] "You may argue, 'I don't care which atom is up.' Perhaps you don't, but nature knows; and ... there isn't any interference. [Conversely] if there is a physical situation in which it is impossible to tell which way it happened, it always interferes; it never fails."

The converse of Feynman's nicely succinct principle is this: If information exists that records the outcome of an 
initially quantum event, that particular aspect of the system ceases to be quantum. This situation never persists, especially with low-mass, low-energy components that quickly reform new quantum states, e.g., the electron emanating as point-source wave after detection in either of two slits in an interference experiment [1].

However, once such an event, no matter how trivial, upsets the smooth expansion of a quantum system within its light cone, that data becomes historical - a small part of the fabric complex fabric classical reality that, if science is to exist at all, must remain intransigently real. Phonons in condensed matter, for example, disrupt the smooth expansion of the location envelopes of individual atoms and thus create historical information: the atom remains locked within its location.

However, it would be a grave error to assume that lowlevel information-generating interactions remove the ability of systems to be quantum in other ways. For example, Fein et al. demonstrated two-slit quantum interference [10] for molecules containing the mass equivalent of over 2,000 carbon atoms. Molecules in this size range store significant historical data about their internal states and are fully capable of evolving new state data even as they behave in a quantum fashion externally.

The critical concept for making sense of such situations is to stop thinking of historical information as a single, uniform entity and instead view it as a fabric of minor histories with wide diversity in data sharing. Those highly isolated from the rest of the universe do not cease to generate unique and statistically irreversible histories, and the histories they create are as valid and non-erasable as any other history. However, their relationship with that larger fabric of history remains problematic if they stay isolated from it for extended periods. So long as their future light cones or, for non-radiating fermionic matter, future Schrödinger expansion cones remain isolated from the rest of the universe, their internal histories remain classically unknown, but not quantum. The world of quantum mechanics is the world of unknown relationships rather than entities.

All of this is another way of emphasizing Hossenfelder's observation that history, once created, does not go away. Thus in the famous case of Schrödinger's cat, the correct answer is that the immensely complex body of the cat, which generated enormous levels of internal information with every moment of its life, is never in a superposition of dead and alive because it is creating its history without any need for involvement from the larger universe. The only aspect of the cat's state still capable of being in question is where a sufficiently isolated cat resides in the spacetime fabric of the universe. Like molecules of over two thousand carbon atoms, the cat may or may not die in the course of some long and very isolated journey, but it can still interfere with itself in its journey a universe that that forgot its existence.

\section{QUANTUM PREDESTINATION AND CATS}

The concept that each unit of independent mass-energy defines its history and that quantum mechanics is the just physics of how those histories interact provides new perspectives on the issue of quantum predestination. The very complexity of the relationships required to implement quantum predestination means that historical data of the forward light cone of the predestination quantum event must be massively and thus historical. The very necessity for complicated integration means there is also no leeway for generating an infinite number of future histories that contain just the right one that results in a correct future detection. While that model of infinite future paths might still work for the location of a highly isolated quantum predestination light, it gives no relief for explaining how the "right" future gets selected.

Yet the problem of non-physical cross-light-cone data transfers and computation remains! How can an appropriate solution be obtained if the soothing manyworlds fantasy of infinite information for asymptotically zero mass-energy cost cannot create the needed futures?

\section{THE BLOCK UNIVERSE PROBLEM}

One temptation is, of course, to invoke a block universe. The problem, in that case, is that such an invocation resolves nothing since a block universe amounts to an assertion that, somehow, it all works out. It does not explain how the coordination for quantum predestination - the complete dependence of a definite future on a much earlier event - comes into existence. Like many worlds, the block universe is a soothing bit of conceptual pablum that provides neither genuine answers nor a path forward for deeper analysis and experimentation.

\section{THE LIGHT CONE AS QUANTUM}

One persistent feature of quantum predestination has been the constraint of the light cone. Systems may develop their histories within that light cone, but the light cone is the one barrier they cannot violate. Even remarkably complex objects can become so "lost" within the $x y z$ space of that light cone that they appear to interfere with themselves to the outside universe, even as their internal histories progress as usual. By quantum rules, they expand within that cone - at light speed for systems that include light, but potentially slowly indeed for massive nonradiating systems with Schrödinger expansion rates. Only when these light cones bump into other entities in other light cones, relationships develop, forcing both entities to assume specific historical stances on how both interpret the collision. Such light cones can even intermingle over a vast range of scales without immediately interacting. Intergalactic photons have light cones of immense size and depth, while atoms in condensed matter batter each other 
with phonons and photons, guarding their spaces and thus limiting the scope of each other's light cones. With each isolated light cone, physics remains the same - the very principle Einstein first recognized in the context of the laws of motion. It is only when the cones of such entities bump into each other that space and time become relative and relational to each other.

The same is true for the quantum relationships of those objects, which become classical and historical only upon interactions that violate their mutual light cones.

Another way of saying this is that each entity generates an expanding envelope of space and time at a rate measured only by its internal clocks. It needs no outside universe, nor any Mach's principle to know how to rotate and feel centrifugal forces. If one component wishes to spin relative to each other, it does so, and experiences the same centrifugal forces as some object whose spin partner is the universe as a whole.

In other words, the spacetime fabric of the universe is not some top-down given but is itself an emergent effect of many tiny quantum systems generating their own time and their own space - a bottom-up universe.

\section{LIGHT CONES AND PREDESTINATION}

The concept of quantum light cones provides a new tool for analyzing the quantum predestination problem. The new tool is this: Bottom-up spacetime does not limit flowof-time in the same fashion as the convenient mathematical abstraction of perfectly smooth, infinitely detailed spacetime. It is lumpy and multi-scale.

The spacetime fabric of condensed matter is tight, detailed, and dominated by Hamiltonian dynamics, with space as smooth and well-behaved as can be found outside black holes, while the mass-starved grains of cosmic voids can grow large beyond any ordinary reasoning.

This lumpiness impacts quantum predestination by enabling a qualified relaxation of time flow [11], though it is a relaxation that is subject to the ever-present constraint that causality - the histories of individual grains cannot be erased or overwritten. Intermixing of large quantum light cones with the tighter fabric of condensed matter allows both to develop causal information based on events that, in the large-grain regions, look simultaneous.

In other words, the simple timelessness of equation transformations in quantum mechanics is not an accident but a reflection of the deeper reality that the ordering relationship we call time is not fundamental to the largescale universe. Big time instead arises as a consensus relationship in complex hierarchies of smaller regions, beginning with incomplete and always-somewhat-fuzzy collections of mass and energy called particles. The equations reflect the timelessness of such components when in isolation, away from any need to define time.

\section{CONCLUSION}

The inspections at both ends of the delayed quantum choice experiment do not happen before or after each other. As with Feynman's timeless photon interactions, the two measurements create a single relationship between the two, "seeing" both as happening at the same instant [12]. It is like quantum predestination in setting up how the future unfolds for recording the initial event, but it is also unlike predestination in the sense that the view of the second observer is no more preordained than that of the first. Both quantum relativity of time and special relativity become instances of the light cone protecting history.

\section{ACKNOWLEDGEMENTS}

Special thanks to Roger Duncan and Ronald Green, both of whom contributed to ideas in this paper.

[1] S. Hossenfelder, "The delayed choice quantum eraser, debunked," Backreaction, 30 October 2021.

[2] A. Peruzzo, P. Shadbolt, N. Brunner, S. Popescu and J. L. OltextquoterightBrien, "A quantum delayed choice experiment," Science, vol. 338, p. 634-637, 2012.

[3] T. Bollinger, "Quantum Erasure as Quantum Predestination," TAO Physics, vol. 2021, p. 1031, 31 October 2021.

[4] S. Hossenfelder, "Superdeterminism: A Guide for the Perplexed," arXiv preprint arXiv:2010.01324, 2020.

[5] H. Everett III, "The Theory of the Universal Wave Function," 1956.

[6] A. Peres, "Reversible logic and quantum computers," Physical review A, vol. 32, p. 3266, 1985.

[7] T. Bollinger, "Yuri Manin on Biomolecules as Quantum Computers," TAO Physics, vol. 2021, p. 0205, 05 February 2021.

[8] T. Bollinger, "Biomolecular Quantum Computation," TAO Physics, vol. 2020, p. 1007, 22 October 2020.

[9] R. P. Feynman, R. B. Leighton and M. Sands, Quote from Lectures III 3-3 paragraph 6 and 3-4 paragraph 1 Unknowability causes interference, vol. 3, Caltech, 1963.

[10] Y. Y. Fein, P. Geyer, P. Zwick, F. Kiałka, S. Pedalino, M. Mayor, S. Gerlich and M. Arndt, "Quantum superposition of molecules beyond 25 kDa," Nature Physics, vol. 15, p. 1242-1245, 2019.

[11] T. Bollinger, "On the Impossibility of Keeping Out Eavesdroppers Using Only Classical Physics," TAO Physics, vol. 2006, no. 01, p. 0123, 23 January 2006.

[12] A. Peres, "Quantum Information and General Relativity," Fortschritte der Physik: Progress of Physics, vol. 52, p. 1052-1055, 2004. 